\title{
The Astronomical Low-Frequency Array (ALFA)
}

D. L. Jones ${ }^{1}$, K. W. Weiler ${ }^{2}$, R. J. Allen ${ }^{3}$, M. M. Desch ${ }^{4}$, W. C. Erickson ${ }^{5}$, M. L. Kaiser ${ }^{4}$, N. E. Kassim², T. B. H. Kuiper ${ }^{1}$, M. J. Mahoney ${ }^{1}$, K. A. Marsh ${ }^{1}$,

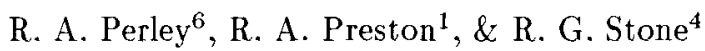

\begin{abstract}
The ALFA mission is designed to map the entire sky at frequencies between approximately 0.3 and $30 \mathrm{MHz}$ with angular resolution limited by interstellar and interplanetary scattering. Most of this region of the spectrum is inaccessible from the ground because of absorption and refraction by the Earth's ionosphere. A wide range of astrophysical questions concerning solar system, galactic, and extragalactic objects could be answered with high resolution images at low frequencies, where absorption effects and coherent emission processes become important and the synchrotron lifetimes of electrons are comparable to the age of the universe.
\end{abstract}

\section{Introduction}

The low frequency window from a few tens of $\mathrm{kHz}$ (just above the local plasma frequency of the interplanetary medium) to a few tens of $\mathrm{MHz}$ (where high resolution observations can be made from the ground) spans three orders of magnitude in frequency, wider than the infrared or ultraviolet windows. It is the last major region of the electromagnetic spectrum still largely unexplored. The goal of the ALFA mission is to image the entire sky at frequencies below $\sim 10 \mathrm{MHz}$ with angular resolution limited only by interstellar and interplanetary scattering.

\section{Science}

The low frequencies to be observed by ALFA are particularly well suited for the detection of "fossil" radio sources and objects emitting coherent radiation. Additional science goals include determining the origin of cosmic rays, mapping the distribution of diffuse $\mathrm{H}$ II in the galaxy, and following the evolution of solar radio bursts. A less specific but equally important goal is exploration. Such a large observational parameter space will be opened for the first time by ALFA that the discovery of completely unexpected sources or processes is likely. This has frequently been the case whenever high resolution observations first became possible in a new part of the spectrum.

\footnotetext{
${ }^{1}$ Jet Propulsion Laboratory, Caltech, Pasadena, CA, USA

${ }^{2}$ Naval Research Laboratory, Washington, D.C., USA

${ }^{3}$ Space Telescope Science Institute, Baltimore, MD, USA

${ }^{4}$ Goddard Space Flight Center, Greenbelt, MD, USA

${ }^{5}$ Univ. of Tasmania, Australia, and Univ. of Maryland, Greenbelt, MD, USA

${ }^{\epsilon}$ National Radio Astronomy Observatory, Socorro, NM, USA
} 


\section{Mission Description}

The array will be composed of 16 very small, spin-stabilized spacecraft in a loosely controlled, three-dimensional cluster $\sim 100 \mathrm{~km}$ in diameter. The entire array along with its deployment and data relay bus is placed in a heliocentric orbit that closely trails the Earth. This allows the array to be established well away from the Earth's noisy radio environment at a relatively low launch cost. The array diameter provides an angular resolution (in all directions) of about 1 arcminute at $10 \mathrm{MHz}$.

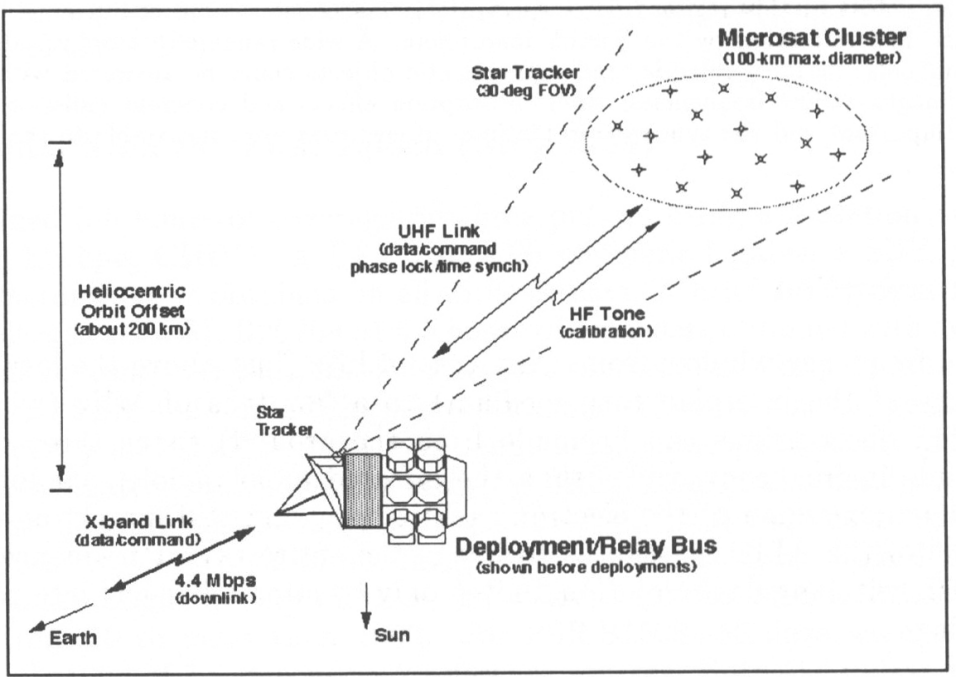

Figure 1. Basic design of the ALFA mission after deployment.

The geometry of the array is monitored by a wide-field star tracker mounted on the deployment bus, which also serves as a data relay to Earth (see figure 1 ). The data will be cross-correlated on Earth for $\sim 10^{3}$ phase centers uniformly distributed across the sky and deconvolution will be carried out for all fields in parallel to allow sidelobes from angularly distant strong sources to be subtracted from the visibility data along with sidelobes from sources within a given field of view. Imaging studies using simulated data have shown that there are no fundamental problems with full-sky imaging with a three-dimensional array such as ALFA if there are enough array elements to provide dense, uniform visibility sampling projected onto all directions simultaneously. S.C. Unwin has developed an algorithm to place array elements on a spherical surface to produce highly uniform sampling in all directions without an over-concentration of short projected baselines.

Acknowledgments. Part of this work was carried out by the Jet Propulsion Laboratory, California Institute of Technology, under contract with the National Aeronautics and Space Administration. KWW \& NEK wish to thank the Office of Naval Research for the 6.1 funding supporting this research. The National Radio Astronomy Observatory is a facility of the NSF, operated under a cooperative agreement by Associated Universities, Inc. 